\title{
Toward a Social Psychophysics of Face Communication
}

\author{
Rachael E. Jack and Philippe G. Schyns
}

Institute of Neuroscience and Psychology, and School of Psychology, University of Glasgow, Glasgow G12 8QB United Kingdom; email: rachael.jack@glasgow.ac.uk

Annu. Rev. Psychol. 2017. 68:269-97

The Annual Review of Psychology is online at psych.annualreviews.org

This article's doi:

10.1146/annurev-psych-010416-044242

Copyright (c) 2017 Rachael E. Jack, Philippe G. Schyns. This work is licensed under a Creative Commons Attribution 4.0 International License, which permits unrestricted use, distribution, and reproduction in any medium, provided the original author and source are credited. See credit lines of images or other third party material in this article for license information.

\section{Keywords}

reverse correlation, social communication, facial expressions, culture, social psychophysics
Abstract
As a highly social species, humans are equipped with a powerful tool for social communication-the face. Although seemingly simple, the human face can elicit multiple social perceptions due to the rich variations of its movements, morphology, and complexion. Consequently, identifying precisely what face information elicits different social perceptions is a complex empirical chal- lenge that has largely remained beyond the reach of traditional methods. In the past decade, the emerging field of social psychophysics has devel- oped new methods to address this challenge, with the potential to transfer psychophysical laws of social perception to the digital economy via avatars and social robots. At this exciting juncture, it is timely to review these new methodological developments. In this article, we introduce and review the foundational methodological developments of social psychophysics, present work done in the past decade that has advanced understanding of the face as a tool for social communication, and discuss the major challenges that lie ahead. 


\section{Contents}

INTRODUCTION ........................................... 270

Social Communication is a System of Information Transmission and Decoding Across a Communication Channel...................... 271

The Human Face Is a Complex, High-Dimensional Dynamic Information

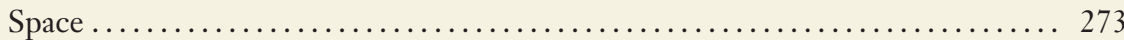

USING THE HUMAN RECEIVER TO UNDERSTAND THE FACE

AS A TOOL FOR SOCIAL COMMUNICATION ................... 274

SOCIAL PSYCHOPHYSICS: DERIVING THE PSYCHOPHYSICAL LAWS

OF SOCIAL PERCEPTION .................................... 275

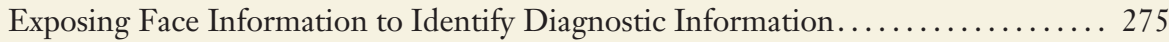

Adding Pixel Noise to Model New Face Information ...................... 277

Generating Face Information from Higher-Order Axes of Information ......... 280

Advantages of a Data-Driven Approach ........................... 283

CONCERNS RAISED ABOUT REVERSE CORRELATION METHODS . . . . . . 284

Why Sample Visual Information? Why Not Instead Increase the Sample Size of Stimuli? .......................................... 284

The Link Between Production and Perception ....................... 284

Analyzing Produced Face Information ............................. 285

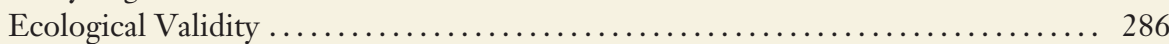

Data-Driven Reverse Correlation Methods Are Atheoretical ................. 287

GUIDING THE USE OF PSYCHOPHYSICAL METHODS ............... 287

Understanding Sender and Receiver Capabilities Across a Communication Channel.................................................. 287

Interactions Between Theory and Data-Driven Approaches................ 288

Exploring the Expanded Social Information Space...................... 289

CONCLUSION ............................................... 289

\section{INTRODUCTION}

As a species, humans have been immensely successful - they have grown rapidly in number, journeyed to and thrived in the most isolated and geographically diverse regions of the planet, and invented medical technologies to control nature (Roberts 2010). One of the main contributing factors to this success is the evolution of humans as socially complex beings; individuals form groups to share labor, knowledge, and resources (Wilson 2012). Consequently, humans are one of the most socially sophisticated species on the planet, engaging in complex interactions to support the functioning of almost every facet of individual lives, including professional, familial, and personal relationships, and wider societal groups such as extended families, neighborhood communities, and workplace environments.

To support these complex social interactions, humans are equipped with a powerful tool-the face (although see also research on voice and body posture, e.g., Atkinson et al. 2004, Belin et al. 2008, Cordaro et al. 2015, Dael et al. 2012, de Gelder 2009, Grezes et al. 2007, Pollick et al. 2001, Roether et al. 2009, Scheiner \& Fischer 2011). As a rich source of information, the human face can elicit myriad immediate social judgments—for example, about identity (e.g., Gauthier et al. 1999, 
Haxby et al. 2000), gender or sex (e.g., Little et al. 2008, Thornhill \& Gangestad 2006), age (e.g., Hummert 2014, Rhodes \& Anastasi 2012), race or ethnicity (e.g., O’Toole et al. 1994, Tanaka et al. 2004), physical health (e.g., Grammer \& Thornhill 1994, Jones et al. 2012), attractiveness (e.g., Perrett et al. 1998, Rhodes 2006), personality traits (e.g., Krumhuber et al. 2007, Oosterhof \& Todorov 2008), sexual orientation (e.g., Freeman et al. 2010, Tskhay et al. 2013), emotions [e.g., Darwin 1999 (1872), Ekman et al. 1969], mental states (e.g., Cunningham et al. 2004, Nusseck et al. 2008), and even religious affiliation (e.g., Rule et al. 2010) and social status (e.g., in pigmentocracies, Telles 2014). In turn, social judgments can have significant consequences for individuals and groups, including influencing employment opportunities (e.g., Johnson et al. 2010), cross-cultural communication barriers (for a review, see Jack 2013), social isolation (e.g., Hawkley \& Cacioppo 2007), voting preferences (e.g., Todorov et al. 2005), and sentencing decisions such as the death penalty (e.g., Eberhardt et al. 2006).

So salient is the face in human society that it has remained the object of fascination, admiration, examination, and debate for several centuries and across broad fields including biology, medicine, art, history, philosophy, psychology, anthropology, and law and, more recently, computer vision and social robotics. One of the most enduring and elusive goals across these fields is to understand how the face elicits the spectrum of social judgments that drives the variety of human behaviors described above. However, understanding how complex face information elicits social perception represents a complex empirical challenge that has largely remained beyond the reach of traditional research methods.

With the development of new research methods from an emerging multidisciplinary scientific culture, the field of social face perception is gaining traction on this question. One increasingly fruitful approach is social psychophysics, which has begun to derive law-like principles of social communication that relate variations of specific face information to specific social perceptions. In turn, such laws have the potential to inform the design of socially aware digital agents (e.g., virtual humans, social robots) that can generate realistic behaviors to communicate with and influence the behavior of different human user groups (e.g., students, children, clinical groups, and cross-cultural groups). In this review, we introduce and review the foundational methodological developments of social psychophysics, present work that has advanced understanding of the face as a tool for social communication, and discuss the major challenges ahead.

\section{Social Communication is a System of Information Transmission and Decoding Across a Communication Channel}

A useful first step in understanding how the face communicates social information is to appreciate the process of communication itself. Like many other forms of communication (e.g., animal mating calls, bacterial quorum sensing), human social communication involves one individual-the sender - transmitting information across a communication channel, which affects the behavior of another individual - the observer (see, e.g., Dukas 1998) or more precisely, as characterized in Shannon's communication model, the receiver (e.g., Shannon 1948). Although the precise definition of communication, including whether the sender or receiver should benefit from the exchange and the role of sender intention, is widely discussed (e.g., Scott-Phillips 2008), we will first focus on the sending and receiving of information between individuals and then discuss the specific aspects of communication that are relevant to understanding the face as a tool for social interaction.

Specifically, communication begins with a message (e.g., I am happy) being encoded as a form of information, such as a dynamic facial expression pattern. This information pattern is then transmitted via a communication channel to the receiver-in this case, the visual information 
pattern of a happy facial expression is transmitted through the medium of light (i.e., photons) to the visual system of another human. For communication to succeed, the transmitted information must first be detectable by the receiver. For example, the laws of optics predict that the fine-grained wrinkles around the eyes of a happy facial expression that project onto the receiver's fixed retina are visible only under specific constraints of the communication channel—in this case, relatively short viewing distances. In contrast, a broad smiling mouth revealing the teeth allows detection over longer viewing distances (Smith \& Schyns 2009) by virtue of its larger size on the retina. We use viewing distance to illustrate how altering the communication channel-i.e., the distance across which the light-based information pattern must be transmitted—can affect the information that is available to the receiver. We discuss other factors that affect the communication channel (e.g., pose or illumination of the sender's face), including sources of noise in the channel (e.g., haze, occlusion of the face), below.

To decode the transmitted information, the receiver must use their prior knowledge- that is, concepts that are biologically, directly, or vicariously acquired—-to extract task-relevant (i.e., diagnostic) information (see, e.g., Schyns 1998). For example, if the receiver aims to detect anger in a given individual (e.g., a spouse or close friend), they might focus their attention specifically on the face regions that reliably indicate anger in this person (e.g., the fixed eyes or tense mouth). The extracted pattern is then compared with a perceptual category that is stored in memory (i.e., a mental representation) and, if it matches, forms the basis of interpreting the message and meaning of the incoming information pattern (e.g., she is angry). Thus, the process of communication involves the human visual system performing the complex task of reducing the varying high-dimensional information impinging the retina (in this example, a complex pattern of face information embedded in a visual scene and transmitted over a variable communication channel) into a smaller set of meaningful categories that are used for adaptive behavior (e.g., spouse, anger, etc.).

In contrast, communication can break down if the transmitted information pattern does not correspond to the receiver's prior knowledge. For example, whereas certain face movements might indicate to a medical professional an underlying neurological condition such as Guillain-Barré syndrome, nonspecialists would derive little meaning from these face-movement patterns. Communication can also break down if the sender and receiver do not associate the information pattern with the same message and meaning. For example, in the United Kingdom, nodding and shaking the head mean yes and no, respectively, but the opposite is true in Bulgaria (see, e.g., Littlewood 2001; for other examples of cross-cultural miscommunication, see Labarre 1947, Morris 1979). Therefore, understanding any system of communication requires identifying which specific information patterns elicit a particular behavior in the receiver, such as the perception that an individual is trustworthy or happy. More specifically, understanding the receiver's stored information patterns - that is, their existing conceptual knowledge or mental representations-and their associated meanings can provide valuable insights into why communication breaks down. For example, comparing the face types associated with the same social message (e.g., trustworthiness or happiness) across cultures could reveal potential sources of miscommunication by showing cultural differences in the expected face movements, morphologies, or complexions.

Understanding the face as a tool for social communication therefore relies on identifying which specific face information elicits different social judgments and, by extension, the different mental representations of individuals in a given culture. However, both endeavors represent a major empirical challenge because (a) the face is a complex visual stimulus in which any dimension of face variation (e.g., movements, morphology, complexion) either individually or in combination could transmit a particular social message, and $(b)$ the detailed mental representations of individuals are generally inaccessible and thus difficult to measure objectively. To further appreciate this challenge, we first detail the complexity of the face as a transmitter of social information before 
describing both classic and recent approaches used to address these challenges; the latter provide new traction on the issue of understanding mental representations and social face perception.

\section{The Human Face Is a Complex, High-Dimensional Dynamic Information Space}

Commensurate with its ability to elicit the perception of multiple intricate social categories, the face comprises several complex dimensions of variation of which three, movement, morphology, and complexion, have mainly been studied. We describe each dimension in turn in this section.

First, the face is equipped with a large number of independent, striated (i.e., voluntarily controlled) muscles that can each be activated individually or in concert to generate observable face movements such as nose wrinkling or smiling. These individual face movements are called Action Units (AUs) in the Facial Action Coding System (FACS) (Ekman \& Friesen 1978), which is an objective system that taxonomizes human facial movements (for chimpanzee face movements, see Vick et al. 2007). FACS identifies over 40 AUs, such as the Upper Lid Raiser (AU5), Nose Wrinkler (AU9), and Lip Corner Puller (AU12), each of which can be combined to create complex AU patterns-i.e., facial expressions. Because facial expressions are dynamic, each individual AU can also be activated with specific temporal characteristics, including acceleration, amplitude, peak latency, and deceleration. Thus, considering all biologically plausible combinations of dynamic AUs - that is, the combinatorics of individual AUs that create multiple patterns and the specific temporal dynamics of each AU—the human face is capable of generating a high number of dynamic facial expressions.

Second, genetic variation such as that within and across ethnic groups in turn produces phenotypic variation in face shape and structure (i.e., morphology; see, e.g., Liu et al. 2012). Historically, the field of anthropometry, which aims to characterize the physical size, form, and function of humans, has used the top-down approach of naturalistic observation to identify up to 31 different dimensions of face variation, including jaw width, forehead height, and interpupillary distance (see, e.g., Zacharopoulos et al. 2016). Over the past three decades, rapid technical developments in face measurement have advanced from laser scanning (e.g., Arridge et al. 1985, Hill et al. 1997, Vetter \& Troje 1997) to high-resolution digital camera capturing (e.g., Hammond \& Suttie 2012, $\mathrm{Yu}$ et al. 2012) combined with specialized face-reconstruction algorithms (e.g., Blanz \& Vetter 1999) to accurately render 3D face morphologies for scientific use [e.g., illumination (e.g., Braje et al. 1998), viewpoint dependence (e.g., Hill et al. 1995, Johnston et al. 2013), and identity (e.g., Leopold et al. 2001, Troje \& Bülthoff 1996)] and computer animation. Thus, based on the several known dimensions of face variation, the number of phenotypically possible face shapes, including those associated with specific medical conditions (for a review, see Winter 1996), is simply huge.

Third, faces also vary in complexion, a dimension that comprises two main dimensions of variation: color, including melanin skin pigmentation and transient pallor or redness, and texture, including wrinkles, scarring, and cutaneous conditions. In the past, melanin skin pigmentation alone has been classified, according to von Luschan's chromatic scale, into 36 different color categories, from very light to olive to very dark (for an example of von Luschan's scale, see Howells 1960); more modern methods using spectrophotometry represent skin color with continuous $\left(\mathrm{L}^{*} \mathrm{a}^{*} \mathrm{~b}\right)$ color values spanning numerous color categories, including purple, blue, pink, and yellow (see, e.g., Swiatoniowski et al. 2013). In addition, skin texture-i.e., relative smoothness—can vary across different scales, from microrelief lines and pitting or raising to larger, deeper markings, each of which can vary in orientation, location, and density, with specific statistics across different face regions (e.g., Lai et al. 2013).

Finally, as a salient platform for social communication, the face is also regularly adorned in a variety of ways, including cosmetics or painting, tattooing, scarring, jewelry, and hair, which are 
typically used to indicate social status or group alliances, or to accentuate beauty by enhancing or camouflaging aspects of face morphology or complexion. As a result of the numerous ways in which the face can vary, the 106 billion humans that have ever been born (Haub 2002) have each possessed a unique face that changes transiently throughout the course of each day and in more permanent ways throughout their lifetime.

As illustrated in this section, the face is a complex and variable visual stimulus that, due to recent technical developments, is only now becoming amenable to precise computational characterizations of its information content and thus to rigorous experimental control. Such developments represent a major turning point in the goal of understanding how the face communicates different social information, and identifying within the complex face information space of movements, morphology, and complexion the specific subspaces that elicit specific social perceptions. Before detailing the advantages of these newly developed methods, it is useful to review both the current state of knowledge in the field and one of the classic approaches to addressing this question.

\section{USING THE HUMAN RECEIVER TO UNDERSTAND THE FACE AS A TOOL FOR SOCIAL COMMUNICATION}

A glance at any face demonstrates how quickly and apparently effortlessly humans extract face information to make a diverse array of social judgments (see, e.g., Bar et al. 2006, Macrae \& Bodenhausen 2000). Commensurate with both the social sophistication of humans and the complexity of the face, the human visual system has coevolved as an efficient and accurate pattern classifier, particularly of social information.

Unsurprisingly then, using the receiver to identify which face information elicits social judgments, as Darwin and many others since have done, has remained one of the most popular methods in the field. One such classic approach involves presenting photographs or videos of real faces and asking receivers to categorize each according to a set of social categories such as basic emotions (e.g., Biehl et al. 1997), personality traits (e.g., Little \& Perrett 2007), or health (e.g., Fink et al. 2006). Based on the receiver's responses and the observable or inferred characteristics of the faces, such as certain facial expressions or the person's ethnicity, age, or sex, the receiver's social judgments are then attributed to one or more of these facial characteristics. For example, male faces tend to be perceived as more dominant than female faces (e.g., Burriss et al. 2007)—i.e., the stimulus category male is associated with higher ratings of perceived dominance than the stimulus category female.

However, the capacity of this approach to understand the face as a tool for social communication relies on its implementation, where traditional use of this method typically provides a relatively narrow account. Specifically, the classic approach described above only associates one category label (e.g., male) with another (e.g., dominant) without showing what specific face information communicates dominance (or sexual dimorphism, the category of male or female). Consequently, it does not address the information ontology of the categorization task-i.e., specific information present in male faces that elicits the social perception of dominance. To understand the face as a tool for social communication, information ontologies are of central importance because they functionally link the stimulus (i.e., specific face information) to behavior (i.e., social perception) and thereby provide a lower bound on the information the receiver's brain must process to produce the behavior in question (Schyns et al. 1998, 2009).

Methodological developments designed to address the limitations of such classic approaches and to characterize the information ontologies of social perception have arisen primarily from the field of psychophysics, which aims to measure the relationship between objectively measurable 
dimensions of a stimulus (e.g., luminance and contrast) and subjective perception (e.g., edge detection). Specifically, psychophysical methods tend to parametrically vary the different dimensions of a stimulus, such as hue and luminance; measure how such variations modulate a given perception, such as color brightness; and derive a law-like model of the relationship using, for example, signal detection theory (Swets et al. 1963) or reverse correlation (Ahumada \& Lovell 1971). Thus, psychophysical laws reveal the critical stimulus information that subtends specific perceptions. Another powerful feature of psychophysical methods is that they tend to be data driven-i.e., stimulus information is sampled agnostically (but typically within the limits of biological possibility or theoretical assumptions, a point we discuss below) and tested against the spectrum of a given perception, which typically extends from no perception to perceptual threshold to saturation (e.g., Snodgrass 1975). Adopting such a data-driven approach and making few a priori assumptions about what information will elicit a specific perception therefore allows for a much broader and potentially more informative exploration of a given space of visual information when attempting to identify what information modulates perception. By extension, psychophysical approaches are thus well suited to understanding what specific face information subtends social perception.

However, as illustrated in the section The Human Face is a Complex, High-Dimensional Dynamic Information Space, the human face is a substantially more complex multidimensional stimulus than those typically used in psychophysical experiments on perception, such as a simple Gabor patch image, which comprises only three dimensions of frequency, contrast, and orientation that can each be easily manipulated. How then can a psychophysical approach be expanded to test complex face-movement patterns, morphology, and complexion information against social perception? In the following sections, we review some of the main psychophysical methods that have been developed to successfully address this question.

\section{SOCIAL PSYCHOPHYSICS: DERIVING THE PSYCHOPHYSICAL LAWS OF SOCIAL PERCEPTION}

As mentioned in the previous section, psychophysical methods typically sample information from a space with few dimensions and test these samples against perception to derive a psychophysical law. Ideally, a psychophysical law of social face perception would detail the contribution of face movements, morphology, complexion, and their combinations to the perception of different social categories. To achieve this, information-sampling methods have taken three main forms: exposing face information in a stimulus, adding noise to a face stimulus, or generating face information from a model. We describe each using examples in the sections below.

\section{Exposing Face Information to Identify Diagnostic Information}

In the field of social face perception, researchers tend to use specific sets of standardized stimuli (e.g., Beaupré et al. 2000, Langner et al. 2010, Lundqvist et al. 1998, Matsumoto \& Ekman 1988) to probe different social perceptions and their associated brain activities (Doty et al. 2014). However, classic use of these stimuli cannot identify the information ontology of a social perceptioni.e., what stimulus information is driving behavior (or brain activity). To address this limitation, the Bubbles method (Gosselin \& Schyns 2001) randomly samples information directly from the stimulus and tests these samples against perception to derive what is typically referred to as a classification image-i.e., an image showing the visual information associated with a given perceptual decision. Figure 1 demonstrates the procedure using the six classic facial expressions of emotion (see Schyns et al. 2007, Smith et al. 2005).

The rationale of the Bubbles method is that the receiver can only categorize the information samples accurately when they comprise task-relevant (i.e., diagnostic) information; for example, 
a
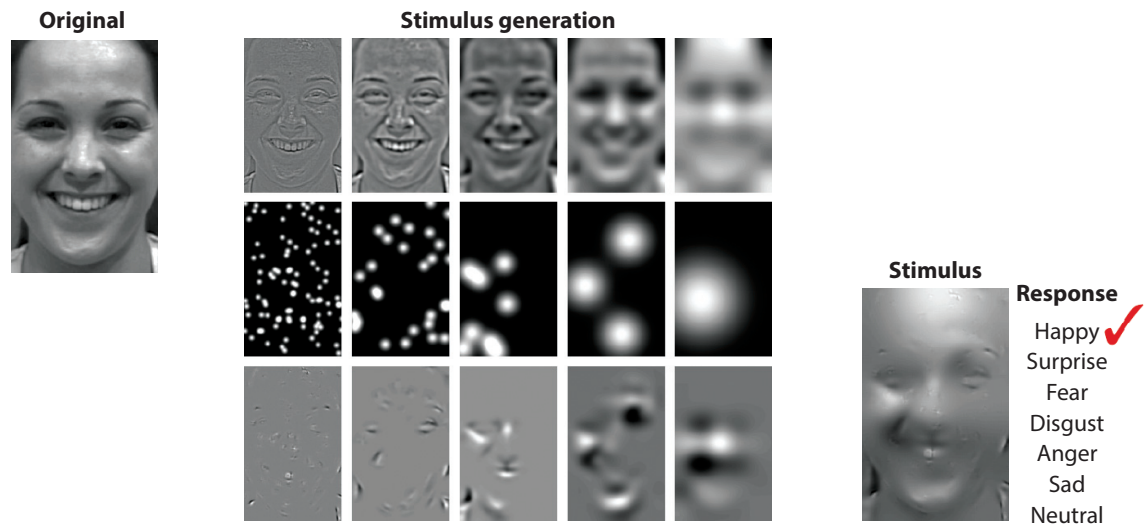

b

Classification images (diagnostic information)

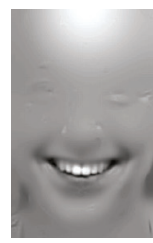

Happy

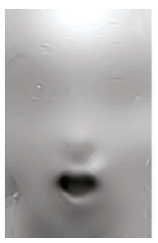

Surprise

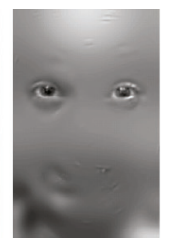

Fear

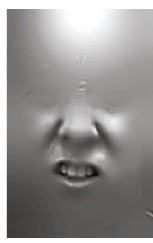

Disgust

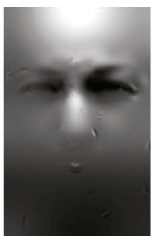

Anger

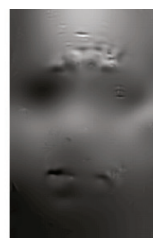

Sad

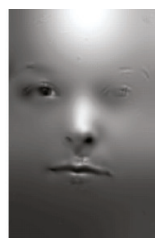

Neutral

Figure 1

Exposing face information. (a) Using the Bubbles method (Gosselin \& Schyns 2001), a stimulus (original) is decomposed into five nonoverlapping spatial frequency bandwidths, each of which are sampled independently with randomly positioned Gaussian windows (the second row of images shows the windows and the third row shows the resulting samples of face information at each bandwidth). The samples of face information (plus a constant, coarser, nonsampled sixth scale not pictured here) are then summed to produce a stimulus (stimulus), which the receiver categorizes according to the six classic emotions (i.e., happy, surprise, fear, disgust, anger, or sad, plus neutral); in this example, the response is happy. (b) To identify the stimulus information required for the accurate categorization of each facial expression of emotion, the image pixels leading to significantly high accuracy at each scale independently are located $(p<0.05$, greater than $75 \%$ correct) and represented in a classification image. Each classification image shows the resulting diagnostic face information for each emotion. Figure adapted from Smith et al. 2005 with permission.

happy is correctly identified because the smiling mouth and the wrinkled eyes are exposed. In contrast, when the random samples do not comprise task-relevant information, the receiver will tend to make an incorrect categorization. Subsequently, by building the relationship between each sampled pixel location at each spatial frequency band and the receiver's correct and incorrect responses (e.g., using correlation, linear regression, or mutual information; see, e.g., Schyns et al. 2011, van Rijsbergen \& Schyns 2009), the Bubbles method can reveal the face-information ontology of each categorization task. Figure $\mathbf{1} \boldsymbol{b}$ shows the information ontologies for the six classic emotions, which comprise smaller subspaces (sets of pixels) of the original image space. For example, recognition of fear requires subsets of pixels representing the eyes, surprise the open mouth, 
and disgust the wrinkled nose and raised upper lip. Overlap of features between expressions, such as the wide opened eyes in both fear and surprise or the wrinkled corners of the nose in both disgust and anger, predicts their behavioral confusions (Smith et al. 2005). Applying the same method to brain activity data such as electroencephalography sensors or magnetoencephalography sources that each form algorithmic brain networks can also reveal the dynamic flow of these information ontologies in the brain (Ince et al. 2015, Schyns et al. 2007).

Whereas the Bubbles method provides some insight into the content of a receiver's mental representations (i.e., stored information patterns), the information ontology obtained is bound to the actual content of the stimuli used, which can therefore limit the characterization of mental representations. For example, standardized FACS coded facial expressions of fear and disgust systematically elicit significantly lower accuracy in receivers belonging to non-Western cultures (for a review, see Nelson \& Russell 2013), which suggests that these particular stimuli do not comprise an information ontology that is close enough to the cultural receiver's mental representations to enable typical recognition performance. An alternative approach to characterizing mental representations is to sample and test face information outside the limits of a specific set of stimuli. How can such information be created? In the following section, we illustrate one method designed to address this question by, seemingly paradoxically, adding pixel noise to a face stimulus.

\section{Adding Pixel Noise to Model New Face Information}

As shown in Figure 2, adding noise such as random grayscale pixel values (Figure $2 \boldsymbol{a}$ ) to a neutral face image can create a perceptively different stimulus. In this example, the receiver categorizes the stimulus as expressive (e.g., of anger) if the random pattern formed by the neutral base face plus noise corresponds (by chance) with their mental representation of that facial expression. If it does correspond, then the white noise template on this trial contains information that corresponds with this receiver's mental representation of a specific facial expression (anger). Thus, to visualize the information content of the receiver's mental representations of different facial expressions (e.g., the six classic emotions), each set of noise templates associated with each emotion category response can be averaged to compute classification images. In this case, the classification image is then added to the neutral base face to visualize how the pattern alters the face. The two faces in the center show the resulting representations of sad and anger for individual receivers. Figure $2 a$ also shows the results from individuals of different cultures (i.e., Western, East Asian). Specifically, Westerners required information to be added to both the eye and mouth regions to perceive emotions, whereas East Asians required much more information to be added to the eyes, for example by changing gaze direction (see Jack et al. 2011). A similar approach using structured (Gabor) noise (Figure $2 \boldsymbol{b}$ ) is used to generate local multiscale random contours on an age-neutral face to estimate, in older and younger adults, the mental representations that underlie their judgments of the chronological age of a face (van Rijsbergen et al. 2014).

Adding noise with more or less intrinsic structure has the advantage of creating for the receiver face information that is not limited to the parameters of an underlying stimulus; this approach also derives the information ontology objectively in a bottom-up manner using the receiver's perceptual response (see the sidebar, Using Noise to Characterize a System; Figure 3). In social psychology, this approach has been used successfully to reveal cultural differences in the mental representations of facial expressions of emotion (Jack et al. 2011), aggression in the mental representations of faces of ethnic out-group members amongst prejudiced individuals (Dotsch et al. 2008), the unattractiveness of newly learned female faces in happily partnered males (Karremans et al. 2011), and the homogenization of older adult faces in the mental representations of younger adults (van Rijsbergen et al. 2014). In visual cognition, this approach has also been used fruitfully 
Adding noise: 2D pixels

a White noise
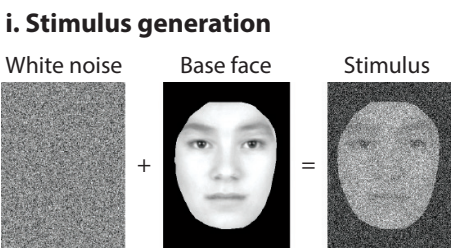

Response

Happy

Surprise

Fear

Disgust

Anger

Sad

ii. Analysis (noise parameters; emotions)

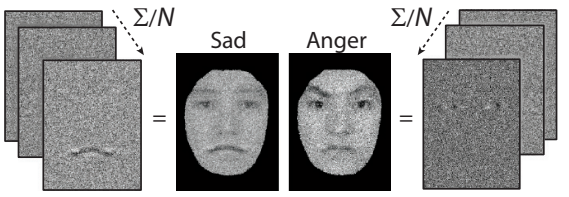

iii. Mental representations

Happy Surprise Fear Disgust Anger Sad

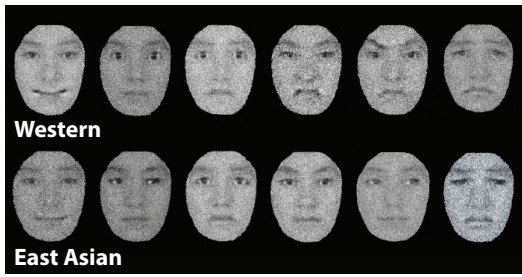

b Structured (Gabor) noise

i. Stimulus generation

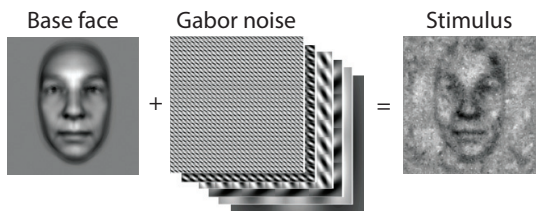

ii. Task: age categorization, 20-35 years
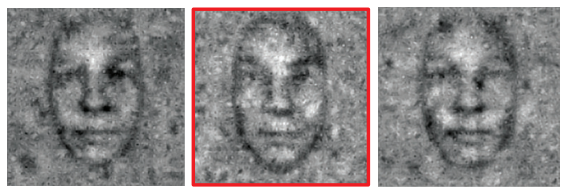

iii. Mental representations

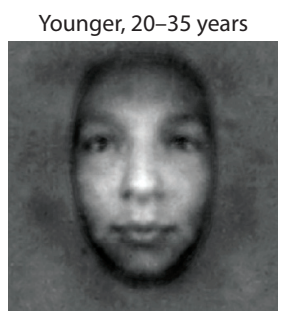

Older, $60-80$ years

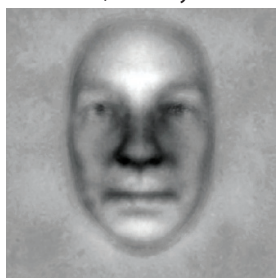

Figure 2

Adding noise to a face. We illustrate two such approaches using $(a)$ white noise and $(b)$ structured (Gabor) noise. $(a, i)$ Adding noise to a neutral base face creates a perceptively different stimulus, which the receiver categorizes according to the six classic emotions if the pattern formed in the stimulus corresponds with their mental representation of that facial expression (in this case, anger). (a, ii) To identify the face information associated with the perception of each emotion, we compute a classification image by averaging the set of noise templates associated with each emotion category response (in this case, sad, outlined in red, and anger, outlined in green) and add it to the base face (central faces). ( $a$, iii) Each face shows a model of an individual receiver's mental representations of each of the six facial expressions of emotion in two cultures: Western (top row) and East Asian (bottom row). Panel adapted from Jack et al. 2011 with permission. (b) Structured (Gabor) noise can also be used to generate local multiscale contours on a neutral base face to identify which face information subtends judgments of chronological age. Panel adapted from van Rijsbergen et al. (2014) with permission.

to estimate mental representations of face identity (e.g., Mangini \& Biederman 2004) and even idiosyncratic letter fonts and smiling faces (Gosselin \& Schyns 2003).

Such methods have provided several substantial knowledge advances in understanding the social perception of faces and, in the best cases, estimating the information content of mental representations, in itself a feat in cognitive psychology. However, these methods also have several drawbacks. First, estimating the relationship between each individual pixel of the noise template and the receiver's social judgments (see the discussion of the first-order kernel in the sidebar, Using Noise to Characterize a System) typically requires several thousand trials per participant because each pixel is considered a statistically independent parameter to estimate (unless assumptions can be made about the relationship between individual pixels, such as local correlations of 


\section{USING NOISE TO CHARACTERIZE A SYSTEM}

Volterra (1930) and Wiener (1958) showed that a system $F$ can be reduced to a sum of subsystems $F_{i}$ if we estimate the kernels (internal functions) $f_{i}$ of each subsystem by sampling white noise and measuring the system's output response. Imagine we instruct a human that they will see white noise images (of $32 \times 43$ pixels) with half of the images comprising a face hidden in the noise. Although their task is to detect the face, only white noise is ever presented. In each experimental trial, the observer uses their expectation of what a face looks like (i.e., their first- and higher-order kernels $f_{i}$ ) and matches this expectation with the incoming white noise. A small positive correlation between the incoming pixel noise and the first-order kernel $f_{1}$ of the brain enables the observer to respond with the perception of a face when the input is only noise. We characterize $f_{1}$ by computing the correlation between each of the $32 \times 43$ pixels (either black or white on each trial) and the detection behavior (face versus no face).

their luminance values). Second, certain face features can be difficult to capture; for example, a deep horizontal wrinkle on the forehead for judgments of older faces would require the unlikely horizontal arrangement of many black and white pixels present on several trials. Rather, a fragment of the horizontal wrinkle is more likely to appear on a given trial, leading the receiver to categorize the face as older. Subsequently, averaging the noise templates associated with older categorizations could result in a horizontal wrinkle across the forehead based on different spatially located wrinkle fragments present in individual trials. Ideally, a full horizontal wrinkle could be revealed using many trials and higher-order analyses of the noise templates-i.e., interactions between two, three, four, or $n$ pixels (see the sidebar Using Noise to Characterize a System, in which pixel order corresponds to kernel order). However, as can be understood intuitively, doing so would be subject to the curse of dimensionality (Bellman 1956) and require a practically impossible number of trials. Nevertheless, first-order analyses, as illustrated in Figure 2, can suggest potential candidates for relevant higher-order features, such as deep horizontal wrinkles, that

Samples

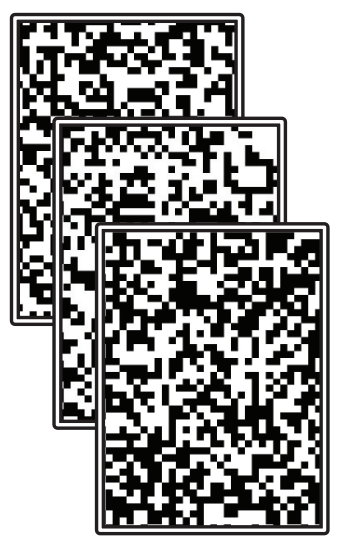

Face detection

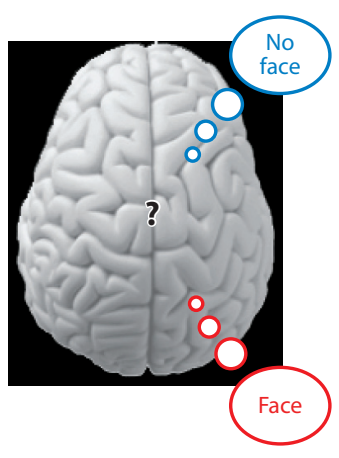

\section{Classification image}
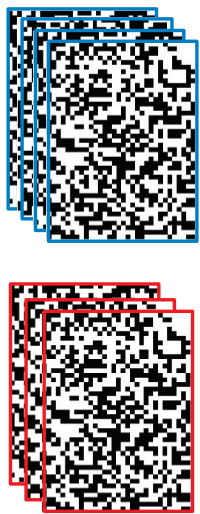

[Noise Sample; Face Detection]

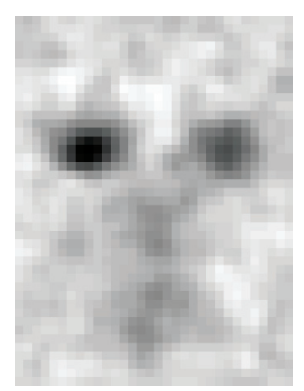

Figure 3

The first-order kernel $f_{1}$, the classification image that estimates the information content of the face prediction of one typical observer (Smith et al. 2012). The classification image depicts the relationship between single-trial random pixel values ( 0 or 1$)$ and face detection behavior (face detected or not), schematized as [Noise Sample; Face Detection]. 
can then be tested for their contribution to social perception (e.g., age judgments) in an explicit experiment.

The above discussion highlights an important consideration for data-driven methods: What information should we sample? For example, rather than sampling individual pixel noise, could we generate information directly from specific higher-order axes of information, such as patterns of face movements, or from multivariate dimensions of 3D face morphology that each constitute a specific hypothesis on the social perception in question (e.g., judgments of personality traits)? The aim of a multivariate approach is to hone in on (and therefore reduce) the relevant parameters for a given perceptual task by capturing the variance of the stimuli in a few axes of information that are tailored to the task at hand. In the following section, we illustrate such an approach using recent methodological developments that can represent and sample higher-order face information such as face movements, morphology, and complexion.

\section{Generating Face Information from Higher-Order Axes of Information}

As described in the section The Human Face is a Complex, High-Dimensional Dynamic Information Space, the individual movements of the face, the AUs, can each be animated and combined to create a variety of dynamic facial expressions. To identify which specific dynamic AU patterns communicate specific social messages to individuals in a given culture, psychophysical methods can be used to directly sample the dynamic information space-i.e., dynamic AUs - and test them against perception. Figure 4 illustrates this method with one example trial.

Compared to classic methods that typically present a limited set of specific facial expression patterns derived from theory, such as FACS-coded facial expressions of emotion, this generative approach allows for a much broader exploration of face-movement patterns as candidates for social communication, including that of emotions (Jack et al. 2012, 2016); mental states such as confusion, interest, and boredom (Chen et al. 2015); and personality traits such as trustworthiness, dominance, and attractiveness (Gill et al. 2014). The above technique of generating face information directly from the higher-order, multivariate information space of dynamic AUs is therefore directly extendable to the multivariate $3 \mathrm{D}$ information spaces of face morphology and complexion. Figure 5 demonstrates the approach using two examples of its application: precisely

Figure 4

Generating face information using 4D Action Units (AUs). (a) In each experimental trial, a Generative Face Grammar platform (GFG) (Yu et al. 2012) randomly selects a subset of AUs (red, green, and blue labels), in this case Upper Lid Raiser (AU5), Nose Wrinkler (AU9), and Upper Lip Raiser (AU10), from a core set of 42 AUs and assigns a random movement to each AU individually using six temporal parameters: onset latency, acceleration, peak amplitude, peak latency, deceleration, and offset latency (labels over the red curve). The GFG then combines the randomly activated AUs to produce a photorealistic facial animation (shown with four snapshots across time). As in Figures 1 and 2, the receiver categorizes the stimulus as socially meaningful (disgust) and rates the intensity of the perceived emotion (strong) when the dynamic pattern correlates with their mental representation of that facial expression. Building a relationship between the dynamic AUs presented in each trial and the receiver's categorical responses produces a mathematical model of each dynamic facial expression of emotion. (b) By including the often-neglected but important dimension of dynamics (for a review, see Krumhuber et al. 2013), such an approach can reveal systematic temporal patterns of face movements: for example, that dynamic facial expressions of emotion transmit face movements in an evolving hierarchy over time, characterized by the transmission of shared face movements early in the time course (e.g., Upper Lid Raiser in both surprise and fear) and different diagnostic face movements later in the time course (e.g., Brow Raiser in surprise and Lip Stretcher in fear; see Jack et al. 2014). 
controlling specific 3D face morphology or complexion associated with different ages, ethnicities, etc. (Figure $\mathbf{5} \boldsymbol{a}-\boldsymbol{c}$ ) and generating novel identities for a reverse correlation approach (Figure $\mathbf{5} \boldsymbol{d}$ ).

At this juncture, it is useful to consider the main differences between generating face information directly from a lower-dimensional multivariate space such as dynamic AUs (e.g., 42 AUs $\times 6$ temporal parameters $=252$ dimensions) and generating information from a higher-dimensional univariate space such as pixel noise (e.g., $380 \times 280$ pixel noise $=106,400$ dimensions). First, using a lower-dimensional space will require much fewer trials to estimate the face information subtending perception. Second, each dimension of the lower-dimensional space, such as $3 \mathrm{D}$ face complexion variations, represents an explicit hypothesis about the information ontology for a

\section{Generating face information: 4D Action Units}

a Modelling dynamic facial expressions

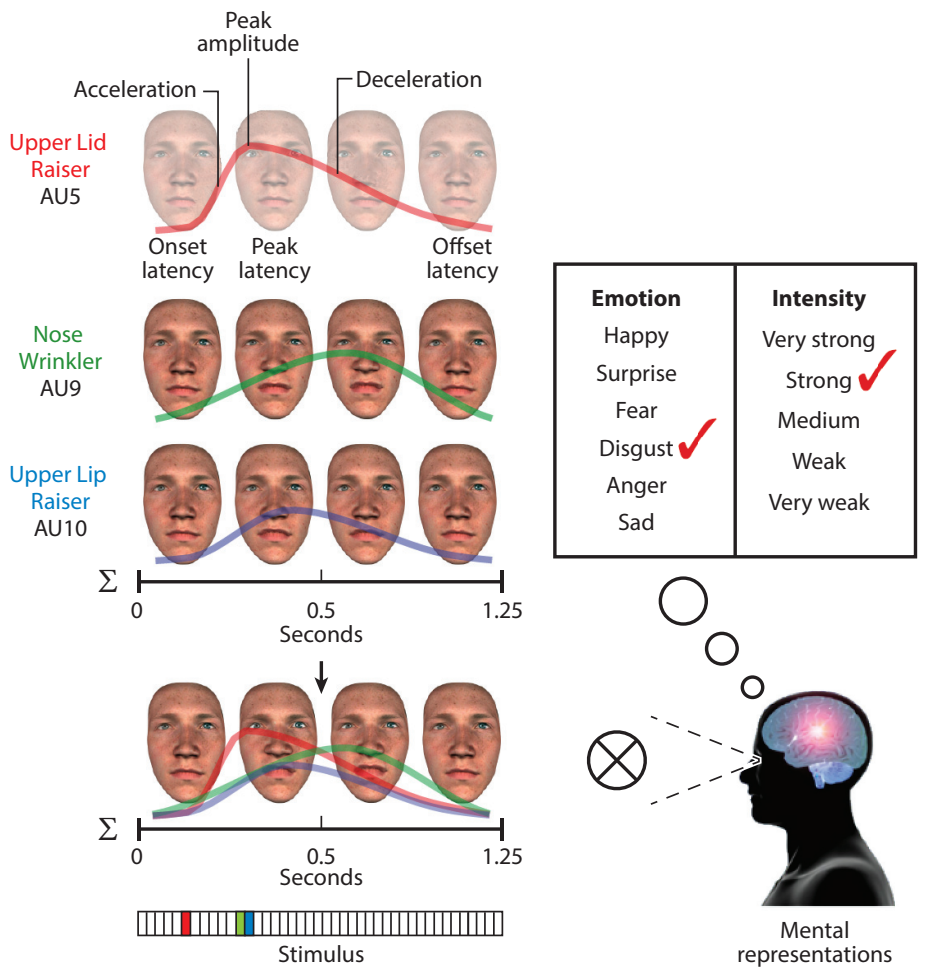

b Hierarchical transmission of face movements over time

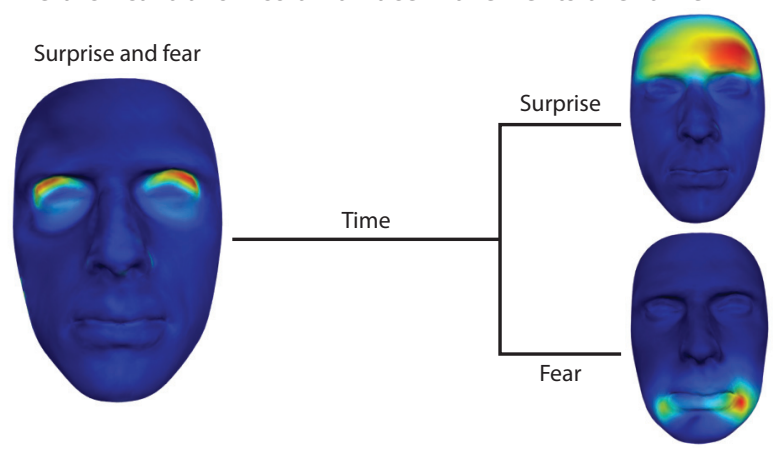


given social perception (e.g., health). Consequently, each resulting psychologically meaningful information subspace-e.g., a specific combination of face color and texture-is bound by the underlying generative information space-e.g., dimensions of 3D face complexion. In contrast, because higher-dimensional noise is not structured, it could in principle reconstruct any face information with sufficiently many trials. However, the curse of dimensionality imposes practical

\section{Generating face information: 3D vertices}

a Morphology ( $x, y, z$ vertices)

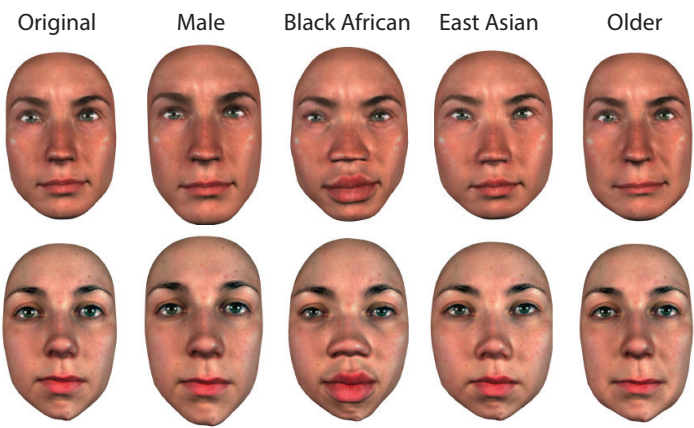

b Complexion $\left(L^{*} \mathrm{a} * \mathrm{~b}\right)$
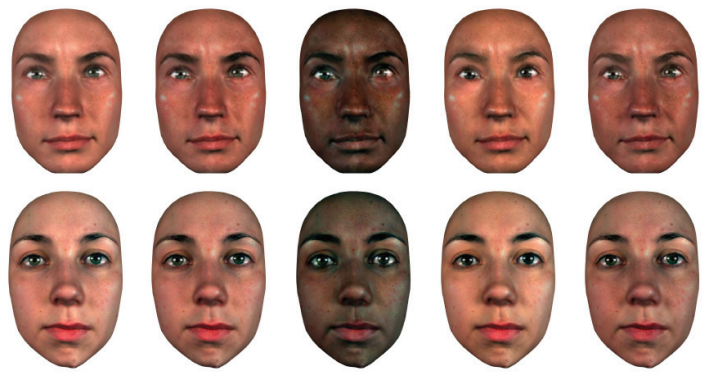

C Morphology and complexion
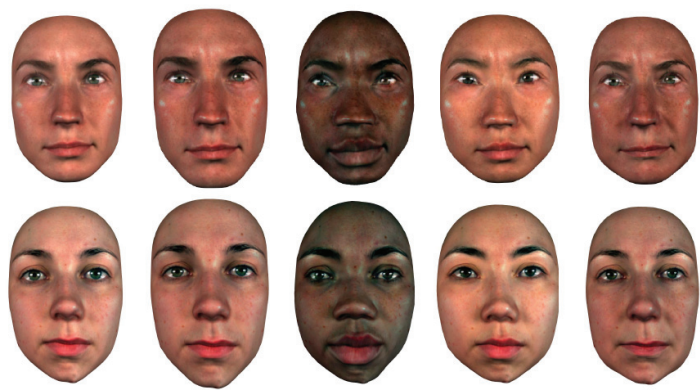

d Task: identity
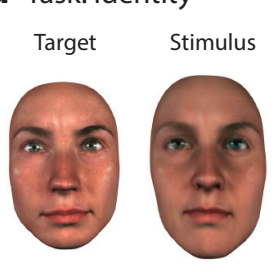

Stimulus

Stimulus

Mental representation
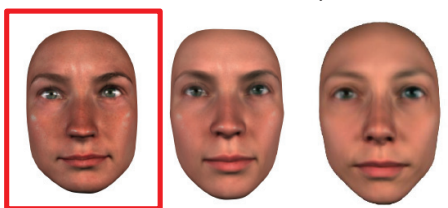
limitations on what can be accomplished with noise, especially when adding dynamics to model face movements or color for complexion. Thus, when designing an experiment based on such psychophysical information-sampling methods, it is critical to carefully consider the axes of information that should be sampled because each axis (e.g., face movements) is an explicit hypothesis on the information thought to be relevant for a given social perception and will thus bound the models that can be derived.

In the above sections, we have demonstrated three main approaches using the classic reverse correlation method in psychophysics to understand what face information drives social perception. We now discuss the strengths of this approach before addressing some concerns raised about its use.

\section{Advantages of a Data-Driven Approach}

Using a data-driven approach provides several advantages. First, psychophysical methods tend to sample information with few a priori assumptions about what face information subtends social judgments. Such an approach makes intuitive sense for the practice of objective investigation and is particularly relevant when studying unfamiliar groups such as different cultures or clinical populations in which existing knowledge is insufficient to provide useful guidance. Consider the scenario of identifying which female physiques are considered desirable in sub-Saharan African cultures. Showing photographs of Coco Chanel or Kate Moss-both Western icons of beauty-to individuals in these cultures would not reveal the sought-after information ontology. Without a more objective, data-driven approach, the female body shapes that are considered desirable in sub-Saharan African cultures-traditionally, clinically obese women-might never even be considered by the Western experimenter (and vice versa). Instead, one approach would be to sample the multivariate variations of 3D human bodies against perceptions of beauty in both cultures (e.g., Mautz et al. 2013). Such an approach could thus be instrumental in bridging the apparently large knowledge gap in psychology arising from the long tradition of empirical and theoretical approaches focused on WEIRD (Western, educated, industrialized, rich, and democratic) cultures (Henrich et al. 2010).

Second, a well-designed information-sampling space, such as one made up of dynamic AUs, multivariate dimensions of $3 \mathrm{D}$ face morphology, or color (typically $\mathrm{L}^{*} \mathrm{a}^{*} \mathrm{~b}$ space), provides a precise visual information space to build rich information ontologies of a variety of social perceptions. Consequently, the resulting information subspaces can reflect a more comprehensive account

\section{Figure 5}

Generating face information using 3D vertices. This reverse correlation approach has been applied to generate and test (a) 3D face morphology, $(b)$ complexion, and $(c)$ their combinations to $(d)$ model the mental representations of face identity. (a) Each row of faces shows a different face identity (original) that has been transformed using samples of $3 \mathrm{D}$ face morphology variation obtained from four different groups (see the labels above each face), with all other dimensions of face variation (i.e., movement and complexion) held constant. (b) The same procedure is applied to 3D complexion. (c) The 3D face information from panels $a$ and $b$ is combined, similar to other methods used to model face information associated with personality judgments such as trustworthiness and dominance (e.g., Oosterhof \& Todorov 2008). (d) Each novel face stimulus (stimulus) is created by sampling 3D face morphology variation from the target identity's gender, age, and ethnic group (target). In each trial, the receiver selects (red outline) the face stimulus that most resembles the target identity based on memory alone. Established statistical procedures such as regression or mutual information, applied to the selected set of face morphologies, provide an estimate of the receiver's mental representation of the target identity (mental representation). Methods such as these can therefore be used to precisely generate, control, and test $3 \mathrm{D}$ face morphology and/or complexion information subtending judgments of identity (e.g., family resemblance), gender, ethnicity, age, and so on. 
of the contribution of face information characterized in quantitative terms, which can support precise, objective analysis and comparison, for example across cultures.

Third, once they are identified, information ontologies behave in a law-like manner. Diagnostic information such as the specific dynamic AU patterns of a positive surprise facial expression in East Asian culture (Jack et al. 2016) could be applied to other East Asian face identities to communicate this social message effectively in this culture. Therefore, one of the main and immediate benefits of developing such generative models is their direct transference to the digital economy via digital agents. For example, social robots and virtual humans are increasingly used in mainstream society to serve a variety of functions, including health care intervention, intelligent tutoring (e.g., social skills training for doctors), companionship, and entertainment (e.g., via the gaming industry) (see, e.g., Morency et al. 2015). Developing and applying such formalisms of face signaling to the design of socially interactive digital agents would allow the generation of realistic and culturally sensitive behaviors with reliable impact on a human user's social judgments (see, e.g., Vinciarelli et al. 2009).

Fourth, the methods outlined above are generic and can thus be used to sample any objectively measureable information space, whether visual, auditory, tactile, olfactory, or gustatory, and to test against almost any form of receiver response, including corresponding sensory perceptions, neural activity (Schyns et al. 2009; for a review, see Wu et al. 2006), or more complex cognitions and behaviors such as approach and avoidance patterns. Sampling combinations of these information spaces can thus further inform the interactive contributions of different sources of information in eliciting a range of behaviors.

\section{CONCERNS RAISED ABOUT REVERSE CORRELATION METHODS}

A number of concerns are often raised when psychophysical methods are used to explore social (i.e., high-level) perception. We address these concerns in the following sections.

\section{Why Sample Visual Information? Why Not Instead Increase the Sample Size of Stimuli?}

One argument against controlling high-level visual information, as described in this review, is that what can be achieved with reverse correlation methods could also be achieved, in principle, by increasing the number of stimuli used in an experimental design. After all, a typical experimental design implicitly samples visual information by sampling a finite set of stimuli assumed to comprise the visual categories of the design (e.g., classic facial expressions of emotion). If this sampling could be infinite, then by definition it would comprise all the dimensions of variance that can be found in naturalistic stimuli. There is one major flaw with this argument, which makes a practical consequence necessary: Stimulus sets are finite because experimental time is finite. Thus, we cannot test a posteriori all possible dimensions of variance, having collected all possible behavioral or neural responses to all possible stimuli. Instead, increasing the fixed number of stimuli inevitably samples information in an implicit, uncontrolled manner. Consequently, we cannot derive the information ontology of a categorization (including social categorizations) unless we explicitly control and sample visual information.

\section{The Link Between Production and Perception}

A commonly raised point is that reverse correlation methods measure the perception but not the production of face information and thus the resulting models of information ontology might only represent a subjective perceptual experience that does not reflect what is actually transmitted by 
the sender. In addressing this concern, it is worthwhile to revisit some fundamental principles, first of general communication and then specifically of visual perception.

First, such concerns imply that the information pattern transmitted by the sender (e.g., a facial expression of anger) is uncorrelated, either in large part or wholly, with the information patterns that elicit perceptions in the receiver (i.e., the information subset, diagnostic information for anger); in other words, perception and production are two independent processes. Instead, as discussed extensively in human (e.g., Scott-Phillips 2008), animal (e.g., Dukas 1998), and humanmade (e.g., Shannon 1948) communication systems, the production and perception of information forms a tightly knit symbiotic relationship in which the capabilities of both sender and receiver shape the ontology of the information patterns that are produced and used for communication. Specifically, if the sender transmits an information pattern that is not meaningful to the receiver (i.e., one that does not correspond to the receiver's prior knowledge), communication tends to break down. Consequently, any such ineffective transmissions will tend to become extinct and replaced with one that is meaningful to the receiver. Thus, the production of information is inextricably shaped by the perceptual and cognitive capabilities of the receiver. One relevant observation from signal phylogeny is that a small discrepancy between the production and the perception of information is necessary for signal evolution (e.g., Arak \& Enquist 1993). Thus, precisely identifying and measuring this discrepancy is relevant to further understanding the nature and development of signals for human social communication (although there is also a distinction between signals, cues, and coercion; see, e.g., Dezecache et al. 2013).

Second, long-standing knowledge of human visual perception shows that there is indeed a distinction between what is produced (i.e., transmitted) and what is perceived (i.e., received and decoded). Specifically, the identification of visual events such as objects, faces, or scenes relies only on a subset of the information that is perceptually available to the receiver (i.e., detectable information) (see, e.g., Schyns 1998). Indeed, classic change-blindness experiments provide elegant demonstrations that not all information transmitted in the environment and perceptually available to the receiver is perceived and memorized (e.g., Simons \& Rensink 2005). Classic Bubbles experiments have also shown that not all perceptually available information is necessary for accurate perceptual categorization (e.g., Gosselin \& Schyns 2002). Thus, understanding communication-e.g., of social categories via the face-requires identifying which subset of the transmitted information subtends the perception of a given social category and so does not necessarily require identifying all that is transmitted (e.g., facial dynamics are unlikely to be necessary for categorizations of ethnicity). Understanding why certain face information is not used for perceptual judgments could further inform the social and biological functions of produced face information, particularly if production is voluntary and costly, as is the case for face movements. For example, a facial expression of disgust might include several different AUs, but accurate perception might only rely on a subset of them (e.g., Nose Wrinkler, Upper Lip Raiser). If only two AUs are necessary and sufficient for accurate recognition, what function, if any, do the others serve? Future work comparing produced face information with the subset that subtends perception could address such questions.

\section{Analyzing Produced Face Information}

In research that focuses on facial expressions, one often-cited alternative to using the receiver to characterize their patterns is to record and analyze spontaneously produced facial expressions. Although this approach should provide a useful complement to a perception-based approach, a number of inherent difficulties currently limit its utility.

Facial expression elicitation methods such as film clips (e.g., Gross \& Levenson 1995) or images (e.g., Lang et al. 2005) typically generate complex combinations of internal states at different 
times—for example, a disgusting film could elicit horror, surprise, and anger-with correspondingly complex combinations of face movement patterns over time. How then can this stream of face movements be interpreted? How can the signs of specific internal states (e.g., horror, surprise, or anger) be extracted? Such difficulties are most evident in computer vision, in which automatic detection systems must first be equipped with knowledge of different facial expression patterns to match and categorize incoming information. A common suggestion to decode the complex stream of face movements is to use human receivers-expert pattern classifiers, particularly of social signals - to interpret the produced facial expressions at each time point. Of course, this is exactly the approach of perception-based reverse correlation methods and thus reflects the strength and intuitive simplicity of the approach. Therefore, although analyzing produced facial expressions is clearly a relevant line of investigation, developing a precise knowledge of the dynamic patterns that communicate different social categories is also necessary. One constructive future research direction would be to develop methods that can precisely compare perception-based models of facial expressions with spontaneously produced facial expressions using, for example, automatic decoding of AUs and their temporal parameters.

\section{Ecological Validity}

Another common concern about reverse correlation methods is that the face stimuli created by sampling (e.g., Bubbles), adding (e.g., white noise), or generating (e.g., Generative Face Grammar) information lack ecological validity and that using real, unobscured images, videos, or live humans more closely represents genuine social interactions. Although methods that use such stimuli arguably provide a more authentic scenario (while usually not incorporating ecological conditions such as occlusion), they typically cannot identify the information ontology that drives behavior-a point highlighted above in the review of the classic approach to understanding social face perception. Such arguments highlight the ever-present tension between achieving ecological validity and obtaining meaningful results, a point that must be discussed constructively to successfully guide research. We open this discussion by considering some often-cited alternatives and evaluating what they can deliver.

First, in the spirit of a psychophysical approach, one solution is to select images or videos of real faces or to use live humans who vary in one aspect of the face (e.g., skin color) while others are controlled (e.g., facial expression) and then to measure the receiver's response to this variation in the stimulus set (e.g., ratings of attractiveness). However, each real face is a unique identity that necessarily comprises a specific morphology (i.e., shape and structure) that will elicit specific social judgments such as attractiveness, trustworthiness, and dominance (e.g., Oosterhof \& Todorov 2008). Consequently, the resulting stimulus set of real faces will inevitably include uncontrolled socially relevant variations that could confound results unless they are fully randomized. Resolving this issue using a finite set of images, videos, or live humans represents a substantial, if not impossible, practical challenge.

A second, often-suggested solution to the problems of using images or videos of real faces or using live humans is to track the receiver's eye movements to study which samples of information are extracted from the stimulus to resolve a given task such as interpreting facial expressions. However, eye movements alone can only index the information in the stimulus that the receiver has fixated and cannot show which information in the fixated content is effectively used for the task (i.e., the diagnostic information). For example, task-relevant information is often extracted extrafoveally (e.g., Blais et al. 2008, Eriksen \& James 1986), and not all fixations made during the task are necessary for accurate perception (Hsiao \& Cottrell 2008). To address some of these 
limitations, new eye-tracking methods that separate foveal versus extrafoveal information (e.g., Miellet et al. 2011) or combine eye-tracking data with statistical modeling techniques (e.g., Jack et al. 2009, Peterson \& Eckstein 2012) provide the required link between stimulus information and behavior to reveal the diagnostic information. Nevertheless, the use of images, videos, or live humans cannot be used to characterize the information ontology of social perception because these real face stimuli comprise fixed information sources that cannot be flexibly manipulated to correspond to a receiver's mental representations.

\section{Data-Driven Reverse Correlation Methods Are Atheoretical}

Data-driven reverse correlation approaches are often described as atheoretical because they make few a priori assumptions about what information will elicit a specific perception. Put more accurately, such methods fundamentally acknowledge that the subspace representing the information ontology of a social category is currently unknown. For example, are judgments of trustworthiness based only on face morphology, or do face movements and complexion also play a role? What about judgments of health or social status? Thus, each dimension of the sampling space (e.g., face movements, morphology, and complexion) in fact forms an explicit hypothesis about the information that is potentially relevant for the social category considered (e.g., trustworthiness, health, social status).

Consequently, the initial challenge of using these methods is to design an information-sampling space that can accurately capture the information ontology for the social category considered. The second challenge is the potential combinatorial explosion of the sampling space used to test against social perceptions - consider, for example, the vastness of a space comprising all variations of face movements, morphology, and complexion. To address these challenges of accuracy and tractability, a creative exercise combining both theoretical and empirical knowledge must be undertaken, first to develop a plausible set of dimensions to sample (e.g., multivariate facial movement patterns to investigate emotion communication) and later to explore how other dimensions (e.g., skin color) might modulate perception. Thus, by using theoretical and empirical knowledge to design and test specific information spaces, the results of data-driven approaches can in turn contribute to the development of existing theories and generate new conceptual advances, thereby both fostering and benefitting from a symbiotic theory-data relationship. In the following sections, we describe some ways to guide the application of psychophysical methods to the high-dimensional and complex stimuli that are typically used in the field of social perception.

\section{GUIDING THE USE OF PSYCHOPHYSICAL METHODS}

\section{Understanding Sender and Receiver Capabilities Across a Communication Channel}

The initial challenge of applying reverse correlation methods is to design an information-sampling space that could accurately capture the information ontology for a given social category while keeping the problem tractable. In our view, this problem remains possibly the most difficult to solve and still requires considerable creative inputs.

In defining such an information space, the most obvious consideration is the physical constraints of the sender and receiver: the human face and visual system, respectively. In the section The Link Between Production and Perception, we discussed briefly the constraint that the information transmitted by the sender must be at least detectable by the receiver. For example, 
certain colors and contrasts cannot be distinguished by the human eye, and detailed information is visible only at relatively short viewing distances (see, e.g., Palmer 1999). Thus, aside from the general biological constraints of the human visual system (e.g., the density and functional nature of retinal receptors), one of the major factors affecting receiver capabilities is the communication channel itself: the medium of light across which visual information is transmitted. Similar to the face, the communication channel is itself a space comprised of different dimensions: distances, illumination, pose, and forms of noise such as occlusion and environmental haze, each of which can impact receiver capability. For example, at short viewing distances all six classic emotionshappy, surprise, fear, disgust, anger, and sad - can be accurately identified from the face based on the perceptual availability of specific face-movement patterns (Smith et al. 2005). However, as viewing distance increases, performance decreases for certain emotion categories because the detailed diagnostic information-e.g., the wide-opened eyes for fear-is no longer perceptually available to the receiver (Smith \& Schyns 2009). Thus, defining the information-sampling space in accordance with the receiver's capabilities depends critically on the constraints of the communication channel that will be used.

With respect to sender capabilities, if the sender is not capable of successfully transmitting information across a specific communication channel-e.g., using the eye whites to communicate fear across a long viewing distance-or if the face information is biologically implausiblee.g., extremely rapid muscle movements, highly saturated colors, or extremely large eyes or tiny mouths-then this information could be omitted from the sampling space. One notable exception is the exploration of superstimuli: exaggerated forms of a stimulus that can elicit stronger responses in the receiver than the original stimulus, such as highly conspicuous courtship and aggression displays (see, e.g., Tinbergen 1948), and that contribute to signal evolution (e.g., Arak \& Enquist 1993). Although superstimuli tend to be beyond the sender's current capabilities, characterizing them still involves identifying, within the original stimulus, the information that elicits a specific response in order to create and test exaggerated versions of the stimulus.

\section{Interactions Between Theory and Data-Driven Approaches}

The design of an information-sampling space can also be informed by theoretical considerations. For example, consider the perceptual judgments that might influence mate choice. This behavior likely involves finding an individual who is in good health and has certain desirable behaviors, such as trustworthiness, warmth, or competence. Although good health is probably indicated by genetically determined (i.e., relatively fixed) aspects of the face, such as symmetry and complexion (e.g., Jones et al. 2012), behavioral intentions, such as trustworthiness and warmth, are more likely indicated by voluntarily produced face information such as facial expressions (e.g., Gill et al. 2014).

Thus, the data-driven methods advocated in this review could be used to model the face movement patterns associated with desirable mate behaviors-e.g., trustworthiness, warmth, and competence- thereby reducing the dynamic information space (i.e., dynamic AUs) to a set of dynamic facial expression models. The resulting models could then be combined with other potentially relevant aspects of face information, such as complexion, to derive a bivariate law of social perception relevant for mate choice. Consequently, such an approach can also explore the important question of how voluntary (i.e., face movements) and involuntary (i.e., morphology and complexion) aspects of the face influence the receiver's judgments within an ecological niche. For example, in countries with racial tension, can voluntarily produced facial expressions associated with trustworthiness override the negative stereotype judgments elicited by involuntary facial cues such as skin color? 


\section{Exploring the Expanded Social Information Space}

In this review, we have described some initial practical steps to exploring how combinations of the complex dimensions of face movements, morphology, and complexion elicit social judgments. Beyond face information, such social judgments could also be influenced by other factors, such as context, the presence (or absence) or other nonverbal information (e.g., body movements or vocalizations), and the communication channel (e.g., short or long viewing distances). As with the dimensions of face variation, each additional potential source of variation nonlinearly increases the complexity of understanding social perception. Thus, although each of these factors might contribute to social perception in some way, it is simply practically impossible to include them all in a full reverse correlation design.

One tractable solution to this problem is to initially model the dimensions that are likely to be most relevant for a given social perception (e.g., face morphology for attractiveness) before adding other potentially relevant dimensions such as body movements, vocalizations, context, and differing communication channels. Consequently, researchers can increasingly refine fundamental models of social perception that capture the main sources of variation, including by demonstrating the robustness of social perception under different conditions (e.g., viewpoint invariance of familiar face recognition). However, this refinement requires sampling a defined and objectively measurable information space. Where no such space exists, one must be created in accordance with existing empirical knowledge or theoretical considerations. For example, although context could be highly relevant for certain social perceptions (see, e.g., Van Bavel et al. 2016), it is not yet well defined or easily measured. In fact, context could be one of the most complex dimensions to test because it spans a broad range of factors, including the social function of the interaction (e.g., cooperation, competition), the current emotional state of sender and/or receiver (e.g., celebrating, mourning), the nature and history of their relationship (e.g., healthy professional, tense spousal), and the physical environment (e.g., hospital, church, workplace, school, outdoors). The challenges of formalizing such knowledge so that it is amenable to sampling indicate that context is one of the most difficult problems in understanding what drives behavior, requiring considerable creative inputs.

\section{CONCLUSION}

In this review, we have introduced and reviewed the foundational methodological developments of social psychophysics - a data-driven approach that can estimate the information ontology subtending social perceptions - with a specific focus on face information. In doing so, we have also highlighted the main strengths of this approach, particularly in addressing the limitations of classic approaches in understanding the face as a tool for social communication, one of the longeststanding and most elusive goals in psychology and other fields such as computer vision.

In looking to the future, it is important to consider the historical trajectory of the fields of psychophysics and social face perception and the more recent development of social psychophysics [a term coined by Davis (1963)]. Psychophysics is one of the oldest fields in psychology and long preceded the classic face recognition studies performed in the mid-twentieth century. However, its application to social face perception has developed substantially within the past decade, made possible by rapid advances in technology, such as $3 \mathrm{D}$ scanning of faces and computational manipulations of stimuli. Creating stimuli with precise characterization and control of face movements, morphology, and complexion would simply not have been possible prior to such advances and in fact would have likely hindered the development of fundamental knowledge arising from these earlier studies. In the absence of sufficient methodological advances, classic theory-driven approaches such as Darwin's [1999 (1872)] and Ekman's (e.g., Ekman et al. 1969) laid the empirical 
and theoretical foundations of understanding the face as a tool for social communication. With rapid advances in technology and methods such as those described in this review, new data-driven approaches can build upon this knowledge to drive the field forward.

This exciting juncture represents a major turning point in the field, and several benefits arise from the arrival of mature social psychophysics. Primarily, social psychophysics can deliver the first formal models - psychophysical laws - that detail the precise contribution of face movements, morphology, and complexion to the variety of social perceptions used for social interactions within and across cultures. Psychophysical laws can support the advancement of knowledge in several ways, including the development of new theories of social face perception that unify fragmented lines of research on face movements, morphology, and complexion, which tend to be studied in isolation. Social psychophysics could also address several outstanding questions, including the in-group facial expression recognition advantage (see, e.g., Elfenbein 2015), encoding of in- and out-group faces (e.g., Adams et al. 2010, Young \& Hugenberg 2012), the other race effect (see Tanaka 2013), and racial stereotyping (e.g., Bijlstra et al. 2010).

The development of such knowledge could have a significant impact in fields beyond psychology, such as computer vision and social robotics, and in wider society. For example, modern society increasingly demands the use of digital agents for a variety of functions, including dayto-day services (e.g., car rental, supermarket shopping, banking); medical and military training; therapeutic interventions; and communication in cyberspace, often across cultures. To be effective, digital agents must generate signals that reliably produce certain behaviors in human users, such as purchasing healthy foods or feeling empathy toward out-group members. Thus, precisely characterizing the algorithms of social perception-i.e., the psychophysical laws - can inform the design of the digital agents of the future and contribute to the development of the digital economy.

Finally, the shift toward a more symbiotic theory-data relationship that bridges the (at least perceived) traditionally disparate scientific cultures of vision science and social psychology is timely for several reasons. Importantly, such relationships can counter the theoretical and empirical stagnation that can arise from the isolation or perceived hierarchy of scientific cultures. For example, theoretical knowledge is sometimes used to judge the value of empirical data, as when theoretically derived AU patterns are used as a gold standard to measure facial expressions despite their low cross-cultural validity. Consequently, embracing a new scientific culture that recognizes the inherent complementarity of these different fields will encourage novel empirical endeavors, such as testing facial expression patterns or social categories not yet represented by existing theories, and facilitate the importation of new methods and knowledge from other fields to address them.

In conclusion, we stand on the brink of a new scientific horizon that promises an era of genuine creativity and the potential to make significant strides in understanding the face as a tool for social communication. Such advances will facilitate the prominent role that social psychology must play in the burgeoning science of social robotics.

\section{SUMMARY POINTS}

1. As one of the most socially sophisticated species on the planet, humans are equipped with a powerful tool for social communication: the face, which, by virtue of its complex variations in movements, morphology, and complexion, can transmit a broad spectrum of social categories from age and sex to social status and trustworthiness. In turn, such perceptions can have significant consequences, including influencing employment opportunities, voting behaviors, and sentencing decisions such as the death penalty. 
2. Understanding which aspects of the face information-i.e., specific variations in face movement patterns, morphology, complexion, or their combination-drive social perceptions has long remained a central question in psychology, philosophy, anthropology, and biology and, more recently, computer vision. However, because the face is a complex, high-dimensional dynamic information space, characterizing the information ontologies of social face perception has remained largely beyond the reach of traditional research methods.

3. New data-driven methods developed in the field of psychophysics can help resolve these issues by deriving law-like principles of social communication that relate variations of face information to social perception, thereby revealing the information ontologies of social face perception.

4. Such data-driven psychophysical methods typically sample information that can be transmitted by the sender (e.g., variations of face movements) across a specific communication channel (e.g., long or short viewing distances) and then measure the receiver's response (e.g., perception of emotion).

5. Psychophysical data-driven approaches have several advantages, including enabling a broader exploration of the social information space due to the application of few a priori assumptions about what information drives perception, a precise and formal characterization of the information ontology of social perception, and the capacity to generate information ontologies.

6. Often-raised concerns about data-driven methods include aspects of ecological validity and lack of theoretical grounding.

7. The use of psychophysical methods to understand social perception can be guided by existing empirical knowledge, such as knowledge of sender and receiver capabilities, and theoretical considerations. Thus the field of social psychophysics is well suited to both contribute to and benefit from a mutually beneficial theory-data relationship.

8. The advent of social psychophysics represents an exciting new juncture in the field of human social perception and communication, which has the potential to develop existing theories, generate new conceptual advances, resolve existing questions (e.g., the in-group facial expression recognition advantage, the other race effect, and racial stereotyping), and foster a symbiotic theory-data relationship within a multidisciplinary scientific culture.

9. This framework can also contribute to the development of knowledge that can be transferred to the digital economy via social robots and virtual humans.

\section{FUTURE ISSUES}

1. Because data-driven methods can be applied to any objectively measurable stimulus information space (e.g., auditory, visual) and linked to measurable behaviors (e.g., perceptual categories, neural activity, decisions), social psychophysics methods can be further developed to explore the extended social environment (e.g., body movements, vocalizations, contextual factors) and thus refine knowledge of nuanced and complex social perceptions. 
2. Data-driven methods also make few a priori assumptions about which information subtends behavior and are therefore well suited to examine social perception in different cultures and atypical groups. Consequently, data-driven approaches could make a significant contribution to advancing knowledge in currently underrepresented non-WEIRD and clinical populations.

3. Law-like principles of social communication have the capacity to generate information ontology, which can be transferred to the digital economy via social robots and virtual humans. Consequently, the field of social psychophysics can inform the design of digital agents of the future so that they can generate realistic behaviors that reliably impact human user engagement.

\section{DISCLOSURE STATEMENT}

The authors are not aware of any affiliations, memberships, funding, or financial holdings that might be perceived as affecting the objectivity of this review.

\section{ACKNOWLEDGMENTS}

We thank Larry Barsalou and Simon Garrod for their insightful and constructive comments on an earlier draft of this review. P.G.S. and R.E.J. received support from the Economic and Social Research Council (ES/K00607/X/1). P.G.S. received support from the Wellcome Trust (Senior Investigator Award, UK; 107802) and the Multidisciplinary University Research Initiative/Engineering and Physical Sciences Research Council (USA, UK; 172046-01). R.E.J. received support from the British Academy (UK; SG113332) and the Economic and Social Research Council (UK; ES/K001973/1).

\section{LITERATURE CITED}

Adams RB, Pauker K, Weisbuch M. 2010. Looking the other way: the role of gaze direction in the cross-race memory effect. 7. Exp. Soc. Psychol. 46:478-81

Ahumada A, Lovell J. 1971. Stimulus features in signal detection. 7. Acoust. Soc. Am. 49:1751-56

Arak A, Enquist M. 1993. Hidden preferences and the evolution of signals. Philos. Trans. R. Soc. Lond. Biol. Sci. 340:207-13

Arridge S, Moss JP, Linney AD, James DR. 1985. Three dimensional digitization of the face and skull. 7. Maxillofac. Surg. 13:136-43

Atkinson AP, Dittrich WH, Gemmell AJ, Young AW. 2004. Emotion perception from dynamic and static body expressions in point-light and full-light displays. Perception 33:717-46

Bar M, Neta M, Linz H. 2006. Very first impressions. Emotion 6:269-78

Beaupré M, Cheung N, Hess U. 2000. The Montreal set of facial displays of emotion. Slides, Dep. Psychol., Univ. Quebec Montr.

Belin P, Fillion-Bilodeau S, Gosselin F. 2008. The Montreal Affective Voices: a validated set of nonverbal affect bursts for research on auditory affective processing. Behav. Res. Methods 40:531-39

Bellman R. 1956. Dynamic programming and Lagrange multipliers. PNAS 42:767-69

Biehl M, Matsumoto D, Ekman P, Hearn V, Heider K, et al. 1997. Matsumoto and Ekman's Japanese and Caucasian Facial Expressions of Emotion (JACFEE): reliability data and cross-national differences. 7. Nonverbal Behav. 21:3-21

Bijlstra G, Holland RW, Wigboldus DH. 2010. The social face of emotion recognition: evaluations versus stereotypes. F. Exp. Soc. Psychol. 46:657-63 
Blais C, Jack RE, Scheepers C, Fiset D, Caldara R. 2008. Culture shapes how we look at faces. PLOS ONE 3:e3022

Blanz V, Vetter T. 1999. A morphable model for the synthesis of 3D faces. Proc. Annu. Conf. Comput. Graph. Interact. Techn., 26th, Los Angeles, Aug. 8-13, pp. 187-94. New York: ACM Press

Braje WL, Kersten D, Tarr MJ, Troje NF. 1998. Illumination effects in face recognition. Psychobiology 26:37180

Burriss RP, Little AC, Nelson EC. 2007. 2D:4D and sexually dimorphic facial characteristics. Arch. Sex. Behav. 36:377-84

Chen C, Garrod O, Schyns P, Jack R. 2015. The face is the mirror of the cultural mind. F. Vis. 15:928

Cordaro DT, Keltner D, Tshering S, Wangchuk D, Flynn LM. 2015. The voice conveys emotion in ten globalized cultures and one remote village in Bhutan. Emotion 16:117-28

Cunningham DW, Kleiner M, Bülthoff HH, Wallraven C. 2004. The components of conversational facial expressions. Proc. Symp. Appl. Percept. Graph. Vis., 1st, Los Angeles, Aug. 7-8, pp. 143-50. New York: ACM Press

Dael N, Mortillaro M, Scherer KR. 2012. The body action and posture coding system (BAP): development and reliability. F. Nonverbal Behav. 36:97-121

Darwin C. 1999 (1872). The Expression of the Emotions in Man and Animals. London: Fontana Press

Davis JA. 1963. Intellectual climates in 135 American colleges and universities: a study in "social psychophysics." Sociol. Educ. 37:110-28

de Gelder B. 2009. Why bodies? Twelve reasons for including bodily expressions in affective neuroscience. Philos. Trans. R. Soc. Biol. Sci. 364:3475-84

Dezecache G, Mercier H, Scott-Phillips TC. 2013. An evolutionary approach to emotional communication. 7. Pragmat. 59:221-33

Dotsch R, Wigboldus DH, Langner O, van Knippenberg A. 2008. Ethnic out-group faces are biased in the prejudiced mind. Psychol. Sci. 19:978-80

Doty TJ, Japee S, Ingvar M, Ungerleider LG. 2014. Intersubject variability in fearful face processing: the link between behavior and neural activation. Cogn. Aff. Behav. Neurosci. 14:1438-53

Dukas R. 1998. Cognitive Ecology: The Evolutionary Ecology of Information Processing and Decision Making. Chicago: Univ. Chicago Press

Eberhardt JL, Davies PG, Purdie-Vaughns VJ, Johnson SL. 2006. Looking deathworthy: perceived stereotypicality of Black defendants predicts capital-sentencing outcomes. Psychol. Sci. 17:383-86

Ekman P, Friesen W. 1978. Facial Action Coding System: A Technique for the Measurement of Facial Movement. Sunnyvale, CA: Consult. Psychol. Press

Ekman P, Sorenson ER, Friesen WV. 1969. Pan-cultural elements in facial displays of emotion. Science 164:8688

Elfenbein HA. 2015. In-group advantage and other-group bias in facial emotion recognition. In Understanding Facial Expressions in Communication, ed. MK Mandal, A Awasthi, pp. 57-71. Berlin: Springer

Eriksen CW, James JDS. 1986. Visual attention within and around the field of focal attention: a zoom lens model. Percept. Psychophys. 40:225-40

Fink B, Grammer K, Matts PJ. 2006. Visible skin color distribution plays a role in the perception of age, attractiveness, and health in female faces. Evol. Hum. Behav. 27:433-42

Freeman JB, Johnson KL, Ambady N, Rule NO. 2010. Sexual orientation perception involves gendered facial cues. Personal. Soc. Psychol. Bull. 36:1318-31

Gauthier I, Tarr MJ, Anderson AW, Skudlarski P, Gore JC. 1999. Activation of the middle fusiform 'face area' increases with expertise in recognizing novel objects. Nat. Neurosci. 2:568-73

Gill D, Garrod OG, Jack RE, Schyns PG. 2014. Facial movements strategically camouflage involuntary social signals of face morphology. Psychol. Sci. 25:1079-86

Gosselin F, Schyns PG. 2001. Bubbles: a technique to reveal the use of information in recognition tasks. Vis. Res. 41:2261-71

Gosselin F, Schyns PG. 2002. RAP: a new framework for visual categorization. Trends Cogn. Sci. 6:70-77

Gosselin F, Schyns PG. 2003. Superstitious perceptions reveal properties of internal representations. Psychol. Sci. 14:505-9 
Grammer K, Thornhill R. 1994. Human (Homo sapiens) facial attractiveness and sexual selection: the role of symmetry and averageness. F. Comp. Psychol. 108:233-42

Grezes J, Pichon S, De Gelder B. 2007. Perceiving fear in dynamic body expressions. NeuroImage 35:959-67

Gross JJ, Levenson RW. 1995. Emotion elicitation using films. Cogn. Emot. 9:87-108

Hammond P, Suttie M. 2012. Large-scale objective phenotyping of 3D facial morphology. Hum. Mutat. $33: 817-25$

Haub C. 2002. How many people have ever lived on Earth? Popul. Today 30:3-4

Hawkley LC, Cacioppo JT. 2007. Aging and loneliness: downhill quickly? Curr. Dir. Psychol. Sci. 16:187-91

Haxby JV, Hoffman EA, Gobbini MI. 2000. The distributed human neural system for face perception. Trends Cogn. Sci. 4:223-33

Henrich J, Heine S, Norenzayan A. 2010. The weirdest people in the world? Behav. Brain Sci. 33:61-83

Hill H, Bruce V, Akamatsu S. 1995. Perceiving the sex and race of faces: the role of shape and colour. Proc. $R$. Soc. Lond. Biol. Sci. 261:367-73

Hill H, Schyns PG, Akamatsu S. 1997. Information and viewpoint dependence in face recognition. Cognition 62:201-22

Howells WW. 1960. The distribution of man. Sci. Am. 203:113-30

Hsiao JH-W, Cottrell G. 2008. Two fixations suffice in face recognition. Psychol. Sci. 19:998-1006

Hummert ML. 2014. Age changes in facial morphology, emotional communication, and age stereotyping. In The Oxford Handbook of Emotion, Social Cognition, and Problem Solving in Adulthood, ed. P Verhaeghen, C Hertzog, pp. 47-60. Oxford, UK: Oxford Univ. Press

Ince RA, Van Rijsbergen NJ, Thut G, Rousselet GA, Gross J, et al. 2015. Tracing the flow of perceptual features in an algorithmic brain network. Sci. Rep. 5:17681

Jack RE. 2013. Culture and facial expressions of emotion. Vis. Cogn. 21:1248-86

Jack RE, Blais C, Scheepers C, Schyns PG, Caldara R. 2009. Cultural confusions show that facial expressions are not universal. Curr. Biol. 19:1543-48

Jack RE, Caldara R, Schyns PG. 2011. Internal representations reveal cultural diversity in expectations of facial expressions of emotion. F. Exp. Psychol. Gen. 141:19-25. doi: 10.1037/a0023463

Jack RE, Garrod OG, Yu H, Caldara R, Schyns PG. 2012. Facial expressions of emotion are not culturally universal. PNAS 109:7241-44

Jack RE, Garrod OGB, Schyns PG. 2014. Dynamic facial expressions of emotion transmit an evolving hierarchy of signals over time. Curr. Biol. 24:187-92

Jack RE, Sun W, Delis I, Garrod O, Schyns P. 2016. Four not six: revealing culturally common facial expressions of emotion. 7. Exp. Psychol. Gen. 145:708-30

Johnson SK, Podratz KE, Dipboye RL, Gibbons E. 2010. Physical attractiveness biases in ratings of employment suitability: tracking down the "beauty is beastly" effect. F. Soc. Psychol. 150:301-18

Johnston A, Hill H, Carman N. 2013. Recognising faces: effects of lighting direction, inversion, and brightness reversal. Perception 42:1227-37

Jones AL, Kramer RS, Ward R. 2012. Signals of personality and health: the contributions of facial shape, skin texture, and viewing angle. F. Exp. Psychol. Hum. Percept. Perform. 38:1353-61

Karremans JC, Dotsch R, Corneille O. 2011. Romantic relationship status biases memory of faces of attractive opposite-sex others: evidence from a reverse-correlation paradigm. Cognition 121:422-26

Krumhuber EG, Kappas A, Manstead AS. 2013. Effects of dynamic aspects of facial expressions: a review. Emot. Rev. 5:41-46

Krumhuber EG, Manstead AS, Cosker D, Marshall D, Rosin PL, Kappas A. 2007. Facial dynamics as indicators of trustworthiness and cooperative behavior. Emotion 7:730-35

Labarre W. 1947. The cultural basis of emotions and gestures. F. Personal. 16:49-68

Lai M, I Oruç, Barton JJ. 2013. The role of skin texture and facial shape in representations of age and identity. Cortex 49:252-65

Lang PJ, Bradley MM, Cuthbert BN. 2005. International affective picture system (LAPS): affective ratings of pictures and instruction manual. Rep. No. A-8, Cent. Study Emot. Atten., Univ. Fla., Gainesville

Langner O, Dotsch R, Bijlstra G, Wigboldus DHJ, Hawk ST, van Knippenberg A. 2010. Presentation and validation of the Radboud Faces Database. Cogn. Emot. 24:1377-88 
Leopold DA, O’Toole AJ, Vetter T, Blanz V. 2001. Prototype-referenced shape encoding revealed by highlevel aftereffects. Nat. Neurosci. 4:89-94

Little AC, Jones BC, Waitt C, Tiddeman BP, Feinberg DR, et al. 2008. Symmetry is related to sexual dimorphism in faces: data across culture and species. PLOS ONE 3:e2106

Little AC, Perrett DI. 2007. Using composite images to assess accuracy in personality attribution to faces. Br. F. Psychol. 98:111-26

Littlewood W. 2001. Cultural awareness and the negotiation of meaning in intercultural communication. Lang. Aware. 10:189-99

Liu F, van der Lijn F, Schurmann C, Zhu G, Chakravarty MM, et al. 2012. A genome-wide association study identifies five loci influencing facial morphology in Europeans. PLOS Genet. 8:e1002932

Lundqvist D, Flykt A, Öhman A. 1998. The Karolinska directed emotional faces. CD-ROM, Dep. Clin. Neurosci., Psychol. Sect., Karolinska Inst., Stockholm

Macrae CN, Bodenhausen GV. 2000. Thinking categorically about others. Annu. Rev. Psych. 51:93-120

Mangini M, Biederman I. 2004. Making the ineffable explicit: estimating the information employed for face classifications. Cogn. Sci. 28:209-26

Matsumoto D, Ekman P. 1988. Japanese and Caucasian Facial Expressions of Emotion (FACFEE) and Neutral Faces ( A ACNeuF). Slides, Dep. Psychiatry, Univ. Calif., San Francisco

Mautz BS, Wong BB, Peters RA, Jennions MD. 2013. Penis size interacts with body shape and height to influence male attractiveness. PNAS 110:6925-30

Miellet S, Caldara R, Schyns PG. 2011. Local Jekyll and global Hyde: the dual identity of face identification. Psychol. Sci. 22:1518-26

Morency L-P, Stratou G, DeVault D, Hartholt A, Lhommet M, et al. 2015. SimSensei demonstration: a perceptive virtual human interviewer for healthcare applications. Presented at AAAI Conf. Artif. Intell., 29th, Jan. 25-30, Austin, TX

Morris D. 1979. Gestures, Their Origins and Distribution. New York: Stein and Day

Nelson NL, Russell JA. 2013. Universality revisited. Emot. Rev. 5:8-15

Nusseck M, Cunningham DW, Wallraven C, Bulthoff HH. 2008. The contribution of different facial regions to the recognition of conversational expressions. F. Vis. 8:1.1-23

Oosterhof NN, Todorov A. 2008. The functional basis of face evaluation. PNAS 105:11087-92

O'Toole AJ, Deffenbacher KA, Valentin D, Abdi H. 1994. Structural aspects of face recognition and the other-race effect. Mem. Cogn. 22:208-24

Palmer SE. 1999. Vision Science: Photons to Phenomenology. Cambridge, MA: MIT Press

Perrett DI, Lee KJ, Penton-Voak I, Rowland D, Yoshikawa S, et al. 1998. Effects of sexual dimorphism on facial attractiveness. Nature 394:884-87

Peterson MF, Eckstein MP. 2012. Looking just below the eyes is optimal across face recognition tasks. PNAS 109:E3314-23

Pollick FE, Paterson HM, Bruderlin A, Sanford AJ. 2001. Perceiving affect from arm movement. Cognition 82:B51-B61

Rhodes G. 2006. The evolutionary psychology of facial beauty. Annu. Rev. Psychol. 57:199-226

Rhodes MG, Anastasi JS. 2012. The own-age bias in face recognition: a meta-analytic and theoretical review. Psychol. Bull. 138:146-74

Roberts A. 2010. The Incredible Human fourney. London: A\&C Black

Roether CL, Omlor L, Christensen A, Giese MA. 2009. Critical features for the perception of emotion from gait. F. Vis. 9:15.1-32

Rule NO, Garrett JV, Ambady N. 2010. On the perception of religious group membership from faces. PLOS ONE 5:e14241

Scheiner E, Fischer J. 2011. Emotion expression: the evolutionary heritage in the human voice. In Interdisciplinary Anthropology: Continuing Evolution of Man, ed. W Welsch, WJ Singer, A Wunder, pp. 105-29. Berlin: Springer

Schyns PG. 1998. Diagnostic recognition: task constraints, object information, and their interactions. Cognition 67:147-79

Schyns PG, Goldstone RL, Thibaut JP. 1998. The development of features in object concepts. Behav. Brain Sci. 21:1-17, discussion 17-54 
Schyns PG, Gosselin F, Smith ML. 2009. Information processing algorithms in the brain. Trends Cogn. Sci. 13:20-26

Schyns PG, Petro LS, Smith ML. 2007. Dynamics of visual information integration in the brain for categorizing facial expressions. Curr. Biol. 17:1580-85

Schyns PG, Thut G, Gross J. 2011. Cracking the code of oscillatory activity. PLOS Biol. 9:e1001064

Scott-Phillips TC. 2008. Defining biological communication. 7. Evol. Biol. 21:387-95

Shannon CE. 1948. A mathematical theory of communication. Mob. Comput. Commun. Rev. 5:3-55

Simons DJ, Rensink RA. 2005. Change blindness: past, present, and future. Trends Cogn. Sci. 9:16-20

Smith FW, Schyns PG. 2009. Smile through your fear and sadness. Psychol. Sci. 20:1202-8

Smith ML, Cottrell GW, Gosselin F, Schyns PG. 2005. Transmitting and decoding facial expressions. Psychol. Sci. 16:184-89

Smith ML, Gosselin F, Schyns PG. 2012. Measuring internal representations from behavioral and brain data. Curr. Biol. 22:191-96

Snodgrass JG. 1975. Psychophysics. In Experimental Sensory Psychology, ed. B Scharf, pp. 17-67. Glenview, IL: Scott Foresman

Swets JA, Green DM, Shipley EF, Sewall SE, Wasserman AG. 1963. Signal detection by buman observers. DTIC Doc., Camb. Res. Lab. Electron., Mass. Inst. Technol. https://dspace.mit.edu/bitstream/handle/ 1721.1/52270/RLE_QPR_053_XX.pdf?sequence=1

Swiatoniowski AK, Quillen EE, Shriver MD, Jablonski NG. 2013. Technical note: comparing von Luschan skin color tiles and modern spectrophotometry for measuring human skin pigmentation. Am. 7. Phys. Anthropol. 151:325-30

Tanaka J. 2013. Introduction. Vis. Cogn. 21:1077-80

Tanaka JW, Kiefer M, Bukach CM. 2004. A holistic account of the own-race effect in face recognition: evidence from a cross-cultural study. Cognition 93:B1-9

Telles E. 2014. Pigmentocracies: Ethnicity, Race, and Color in Latin America. Chapel Hill: Univ. N.C. Press

Thornhill R, Gangestad SW. 2006. Facial sexual dimorphism, developmental stability, and susceptibility to disease in men and women. Evol. Hum. Behav. 27:131-44

Tinbergen N. 1948. Social releasers and the experimental method required for their study. Wilson Bull. 60:6-51

Todorov A, Mandisodza AN, Goren A, Hall CC. 2005. Inferences of competence from faces predict election outcomes. Science 308:1623-26

Troje NF, Bülthoff HH. 1996. Face recognition under varying poses: the role of texture and shape. Vis. Res. 36:1761-71

Tskhay KO, Feriozzo MM, Rule NO. 2013. Facial features influence the categorization of female sexual orientation. Perception 42:1090-94

Van Bavel JJ, Mende-Siedlecki P, Brady WJ, Reinero DA. 2016. Contextual sensitivity in scientific reproducibility. PNAS 113:6454-59

van Rijsbergen N, Jaworska K, Rousselet GA, Schyns PG. 2014. With age comes representational wisdom in social signals. Curr. Biol. 24:2792-96

van Rijsbergen NJ, Schyns PG. 2009. Dynamics of trimming the content of face representations for categorization in the brain. PLOS Comput. Biol. 5:e1000561

Vetter T, Troje NF. 1997. Separation of texture and shape in images of faces for image coding and synthesis. 70SAA 14:2152-61

Vick S-J, Waller BM, Parr LA, Pasqualini MCS, Bard KA. 2007. A cross-species comparison of facial morphology and movement in humans and chimpanzees using the facial action coding system (FACS). 7. Nonverbal Behav. 31:1-20

Vinciarelli A, Pantic M, Bourlard H. 2009. Social signal processing: survey of an emerging domain. Image Vis. Comput. 27:1743-59

Volterra V. 1930. Theory of Functionals and of Integral and Integro-Differential Equations. London: Blackie

Wiener N. 1958. Nonlinear Problems in Random Theory. Cambridge, MA: MIT Press

Wilson EO. 2012. The Social Conquest of Earth. New York: WW Norton \& Co.

Winter RM. 1996. What's in a face? Nat. Genet. 12:124-29

Wu MC-K, David SV, Gallant JL. 2006. Complete functional characterization of sensory neurons by system identification. Annu. Rev. Neurosci. 29:477-505 
Young SG, Hugenberg K. 2012. Individuation motivation and face experience can operate jointly to produce the own-race bias. Soc. Psychol. Personal. Sci. 3:80-87

Yu H, Garrod OGB, Schyns PG. 2012. Perception-driven facial expression synthesis. Comput. Graph. 36:15262

Zacharopoulos GV, Manios A, Kau CH, Velagrakis G, Tzanakakis GN, de Bree E. 2016. Anthropometric analysis of the face. F. Craniofac. Surg. 27:e71-e75

\section{RELATED RESOURCES}

Cohen MM. 2006. Perspectives on the Face. Oxford, UK: Oxford Univ. Press

Eckstein MP, Ahumada AJ. 2002. Classification images: a tool to analyze visual strategies. F. Vis. 2:1. doi: $10.1167 / 2.1 .1$

Guilford T, Dawkins MS. 1991. Receiver psychology and the evolution of animal signals. Anim. Behav. 42:1-14

Jack RE, Schyns PG. 2015. The human face as a dynamic tool for social communication. Curr. Biol. 25:R621-34

MacLin OH, MacLin MK, Peterson D, Chowdhry O, Joshi P. 2001. Social psychophysics: using psychophysics to answer "social" questions with PsychoPro. Behav. Res. Methods 41:623-32

Murray RF. 2011. Classification images: a review. F. Vis. 11:2

Yost WA, Fay RR. 2012. Human Psychophysics, Vol. 3. Berlin: Springer 
Annual Review of

Psychology

Volume 68, 2017

\section{Contents}

Eavesdropping on Memory

Elizabeth F. Loftus

Memory: Organization and Control

Howard Eichenbaum

Neural Mechanisms of Selective Visual Attention

Tirin Moore and Marc Zirnsak

Learning, Reward, and Decision Making

John P. O'Doherty, Feffrey Cockburn, and Wolfgang M. Pauli

Reinforcement Learning and Episodic Memory in Humans and Animals:

An Integrative Framework

Samuel 7. Gershman and Nathaniel D. Daw

Social Learning and Culture in Child and Chimpanzee Andrew Whiten

Survival of the Friendliest: Homo sapiens Evolved via Selection for

Prosociality

Brian Hare

Numerical Development

Robert S. Siegler and David W. Braitbwaite...

Gene $\times$ Environment Interactions: From Molecular Mechanisms to

Behavior

Thorbildur Halldorsdottir and Elisabeth B. Binder

The Structure of Social Cognition: In(ter)dependence of Sociocognitive

Processes

Francesca Happé, Jennifer L. Cook, and Geoffrey Bird

Toward a Social Psychophysics of Face Communication

Rachael E. Fack and Philippe G. Schyns

Social Motivation: Costs and Benefits of Selfishness and Otherishness

Jennifer Crocker, Amy Canevello, and Ashley A. Brown

Attitude Strength

Lauren C. Howe and Fon A. Krosnick

How Power Affects People: Activating, Wanting, and Goal Seeking Ana Guinote 
The Psychology of Close Relationships: Fourteen Core Principles

Eli 7. Finkel, feffry A. Simpson, and Paul W. Eastwick

Moving Beyond Correlations in Assessing the Consequences of Poverty

Greg F. Duncan, Katherine Magnuson, and Elizabeth Votruba-Drzal .......

Culture Three Ways: Culture and Subcultures Within Countries

Daphna Oyserman

Learning from Errors

Fanet Metcalfe

Mindfulness Interventions

7. David Creswell.

Hidden Wounds? Inflammatory Links Between Childhood Trauma and

Psychopathology

Andrea Danese and Fessie R. Baldwin

Adjusting to Chronic Health Conditions

Vicki S. Helgeson and Melissa Zajdel

Health Behavior Change: Moving from Observation to Intervention

Paschal Sheeran, William M.P. Klein, and Alexander 7. Rotbman ....

Experiments with More than One Random Factor: Designs, Analytic Models, and Statistical Power

Charles M. Fudd, Facob Westfall, and David A. Kenny

Interactions with Robots: The Truths We Reveal About Ourselves

Elizabeth Broadbent....

\section{Indexes}

Cumulative Index of Contributing Authors, Volumes 58-68

Cumulative Index of Article Titles, Volumes 58-68 658

\section{Errata}

An online log of corrections to Annual Review of Psychology articles may be found at http://www.annualreviews.org/errata/psych 